\title{
Introduction: Transport and land use in childhood
}

\author{
Ronald Buliung \\ University of Toronto \\ ron.buliung@utoronto.ca
}

\author{
Raktim Mitra \\ Ryerson University \\ raktim.mitra@ryerson.ca
}

What is the relationship between childhood, transportation, and land use? What are the social and environmental - along with political, economic, and policy-related factors and forces - that produce transport in childhood? How do we create places where childhood is considered more explicitly in transport and land-use planning? What is the effect of research methods in producing knowledge about the relationship between childhood, transport, and land use? These are some of the questions that shaped the call for papers, "Children and Youth Transport and Land Use: Theory, Method and Applications," for the 2014 World Symposium on Transportation and Land Use Research held in Delft, Netherlands.

The call attracted research articles that were international in scope, though focused primarily on children's mobility in the Global North. Across the articles, childhood is represented as a biomedical and developmental concept - centered on age, stage, and developmental milestones - and as a sociological construct that is understood to have diverse historical, political, sociocultural, economic, and environmental roots. It is also fair to say that the contributors to this issue consider the experiences of childhood to be meaningful and "useful" to the contemporary conversation about city building and the sustainability of transport systems. Using this diverse theoretical conceptualization as a backdrop, what is so urgent about the current state of childhood in the city that warrants attention at conferences, and subsequent development of a collection of articles?

Just as our cities and regions have been fundamentally transformed in their geographical extent, internal design, and spatial structure of flows (information, capital, people) throughout the automobile century, so too have the everyday lives of children and youth. The auto-orientation of childhood in the Global North, which accelerated during the post-World War II era-an era also characterized by accelerated development of far-flung automobile suburbs_-associates with a decline in childhood health. The symptoms of this decline include increased sedentary activity, rising prevalence of obesity in childhood, and the increased early onset of chronic diseases (Janssen and LeBlanc 2010; Shields 2006; Rennie et al. 2005; Trost et al. 2001; Goran 2008). Beyond these health concerns, we might consider that the escorted and/or chauffeured child of today might not become the active traveler of tomorrow (Roberts 1996). Research also suggests that a chauffeured child's environmental acuity and neighborhood environmental knowledge may be limited by automobility (Fusco et al. 2012).

When making the case for transport and land use studies focused on children and youth, we often turn to the changing nature of the school trip. This is due in part to the fact that scholars have access to school travel data, for many different places and historical periods, and also because the school run represents a moment in childhood where we learn how to negotiate and experience transportation,

Copyright 2016 Ronald Buliung and Raktim Mitra

http://dx.doi.org//10.5198/jtlu.2015.915

ISSN: 1938-7849 | Licensed under the Creative Commons Attribution - Noncommercial License 3.0

The Journal of Transport and Land Use is the official journal of the World Society for Transport and Land Use (WSTLUR) and is published and sponsored by the University of Minnesota Center for Transportation Studies. This paper and others from WSTLUR 2014 are also published with sponsorship from WSTLUR and the Institutes of Transportation Studies at the University of California, Davis, and the University of California, Berkeley. 
for an obligatory activity, on a daily basis. Looking across North America and parts of Europe, what we have seen is a steady decline in active school transportation (more specifically, walking and cycling) since the end of World War II (Buliung et al. 2009; McDonald et al. 2011; Pooley et al. 2005). Four of the five papers included in this issue address this problem by examining walking and cycling for school transportation, using case studies from Toronto, Canada (Larsen et al. 2016; Mitra et al.,2016), Rishon LeZion, Israel (Moran et al. 2016), and Helsinki, Finland (Sarjala et al. 2016). More specifically, these papers focus on the potential influence of the neighborhood built environment on active mobility in childhood. Some of these papers emphasize measurement issues, particularly with regard to travel routes (Larsen et al. 2016) and neighborhood characteristics (Sarjala et al. 2016), to improve our understanding of the geography of children's mobility. Others highlight the importance of distinguishing between travel modes when exploring school travel or other aspects of childhood mobility and point out that a "walkable" neighborhood may not always be "bikeable" (Moran et al. 2016).

Situating children's mobility within its historical context is also important; there exists no universal childhood experience, either historically or geographically (Prout 2005). Mitra and his colleagues (2016) provide a historical perspective on the decline in active school transportation between 1986 and 2006 in Toronto, Canada, where they report a potentially declining importance of the neighborhood environment, and perhaps an increasing influence of school-travel distance, in mobility decisions.

Over the past decades, we have also seen an increase in the supervision of children for most things, including transportation (Hillman et al. 1990; Mackett et al. 2007). Active travel (e.g., walking, cycling) presents an opportunity for children to practice independent mobility (Mitra 2013). For the majority of today's children, constant parental supervision can be seen as a barrier that may deprive them from myriad physical, social, and health benefits (Mackett et al. 2007; Mitra et al. 2014). Interestingly, the opposite may be true for those who are on the verge of adulthood (i.e., teenagers), for whom the ability to drive may be considered an important step toward independence from parental supervision and chauffeuring. McDonald et al. (2016) confirm this hypothesis in their study focusing on teenage girls in the US cities of San Diego and Minneapolis. However, the authors found that the geography of activity participation remained somewhat unchanged regardless of licensure.

The publication of this issue is quite timely, as it intersects with data suggesting that the social and economic polarization we are seeing at essentially all scales, from the local to global, is rising among children living in so-called developed economies (Hudson and Kühner 2016). In this issue, the relationship between income and children's transport and land use is directly addressed in the papers by Larsen et al. (2016), Mitra et al. (2016), McDonald et al. (2016), and Sarjala et al. (2016). Arguably, there is more work to be done to understand what is happening at the intersection between poverty, land use, transportation, and childhood. The mediating role of culture and race, as McDonald et al. (2016) begin to unfold in their paper, also remains an important topic for future research.

Advancing a research agenda focused on interactions between modern childhood, transport, and land use is justifiable given a context characterized by declining population health and rising automobile use. Many scholars, some politicians, and practicing planners claim to be interested in changing how we move about, with a view to positively affecting population health, climate change, and the wanton consumption of viable agricultural land for development. If we or they are actually serious about this, then childhood warrants considerable attention as a starting point for planning how we can proceed with the long-term project of changing our urban development and transport practices and cultures. Contributing to the development of evidence to inform an understanding of childhood, transport, and land-use interactions - with a view to ultimately making childhood something that is less exotic and more accepted, standard, and/or considered when we plan and design land use and transport-is, in part, what this issue is about. We hope you enjoy the papers and are similarly inspired to consider children in your future research. 


\section{References}

Buliung, R. N., R. Mitra, and G. Faulkner. 2009. Active school transportation in the Greater Toronto Area, Canada: An exploration of trends in space and time (1986-2006). Preventive Medicine 48: 507-512.

Fusco, C., F. Moola, G. Faulkner, R. Buliung, and V. Richichi. 2012. Toward an understanding of children's perceptions of their transport geographies: (Non)Active school travel and visual representations of the built environment. Journal of Transport Geography 20(1): 62-70.

Goran, M. I. 2008. Energy expenditure, body composition, and disease risk in children and adolescents. Proceedings of the Nutrition Society 56:195-209.

Hillman, M., J. Adams, and J. Whitelegg. 1990. One False Move: A Study of Children's Independent Mobility. London: PSI Press.

Hudson, J., and S. Kühner. 2016. UNICEF Innocenti Report Card 13 Children in the Developed World. Available at: https://www.unicef-irc.org/publications/series/16/ [Accessed May 2016].

Janssen, I., and A. G. LeBlanc. 2010. Systematic review of the health benefits of physical activity and fitness in school-aged children and youth. International Journal of Behavioral Nutrition and Physical Activity: 40.

Larsen, K., R. N. Buliung, and G. Faulkner. 2016. School travel route measurement and built environment effects in models of children's school travel behavior. Journal of Transportation and Land Use 9(2): 1-19.

Makett, R., B. Brown, Y. Gong, K. Kitazawa, and J. Paskins. 2007. Setting children free: Children's independent movement in the local environment. Paper 118, UCL Working Paper Series. Centre for Advanced Spatial Analysis, University College London.

McDonald, N. C., A. L. Brown, L. M. Marchetti, and M. S. Pedroso. 2011. U.S. school travel, 2009: An assessment of trends. American Journal of Preventive Medicine 41:146-151.

McDonald, N., L. A. Merlin, H. Hu, J. Shih, D. A. Cohen, K. R. Evenson, T. L. Mckenzie, and D. A. Rodriguez. 2016. Longitudinal analysis of adolescent girls' activity patterns: Understanding the influence of the transition to licensure. Journal of Transport and Land Use 9(2): 1-20.

Mitra, R. 2013. Independent mobility and mode choice for school transportation: A review and framework for future research. Transportation Reviews 33(1): 21-43.

Mitra, R., G. E. J. Faulkner, R. N. Buliung, and M. R. Stone. 2014. Do parental perceptions of the neighbourhood environment influence children's independent mobility? Evidence from Toronto, Canada. Urban Studies 51 (16): 3401-3419.

Mitra, R., E. M. Papaioannou, and K. M. N. Habib. 2016. Past and present of active transportation: An explanation of the influence of the built environment in Toronto, Canada, from 1986 to 2006. Journal of Transport and Land Use 9(2): 1-17.

Moran, M., P. Plaut, and O. Baron-Espel. 2016. Do children walk where they bike? Exploring built environment correlates of children's walking and bicycling. Journal of Transport and Land Use 9(2): $1-23$.

Pooley, C. G., J. Turnbull, and M. Adams. 2005. The journey to school in Britain since the 1940s: continuity and change. Area 37: 43-53.

Prout, A. 2005. The Future of Childhood. London: Routledge.

Rennie, K. L., L. Johnson, and S. A. Jebb. 2005. Behavioural determinants of obesity. Best Practice \& Research Clinical Endocrinology \& Metabolism 19: 343-358.

Roberts, I., 1996. Children and sport: Walking to school as future benefits. BMJ 312: 1229.

Sarjala, S., A. Broberg, and A. Hynynen. 2016. Children and youth transport in different urban mor- 
phological types. Journal of Transport and Land Use 9(2): 1-17.

Shields, M. 2006. Overweight and obesity among children and youth. Statistics Canada: Health Reports 17: $27-42$.

Tranter, P., and J. Whitelegg. 1994. Children's travel behaviours in Canberra: Car dependent lifestyles in a low-density city. Journal of Transport Geography 2: 265-273.

Trost, S. G., L. M. Kerr, D. S. Ward, and R. R. Pate. 2001. Physical activity and determinants of physical activity in obese and non-obese children. International Journal of Obesity and Related Metabolic Disorders 25: 822-29. 